\title{
Use of Topical Lidocaine, Diphenhydramine Hydrochloride, Nystatin, and Gabapentin Swish in Treatment for Post-Radiation Neuropathy and Oral Mucositis
}

\section{TO THE EDITOR:}

The pain caused by cancer treatment-related oral mucositis is often described as the most excruciating symptom (1). Frequently, it leads to reduced ingestion, malnutrition, and sometimes postponement or withdrawal of the therapy (1). For health care providers, adequate pain treatment is a major challenge. Treatments that have varying degrees of success include antibiotics, antifungal, antivirals, opioids, benzydamine oral rinse, and palifermin $(2,3)$. Topical pain management is invariably administered in most patients due to its favorable risk-benefit profile and adjuvant role $(2,3)$. However, topical treatment of mucositis pain today is based on empiricism and not on scientific evidence $(2,3)$. We report the use of a novel topical mixture of lidocaine, diphenhydramine hydrochloride, nystatin, and gabapentin along with a course of oral opioids for radiation-induced intractable oral pain.

\section{Case Presentation}

A squamous cell carcinoma of the right base of the tongue was diagnosed in a 76-year-old woman. She underwent initial chemotherapy and radiotherapy leading to temporary remission. However, 2 years later the tumor reoccurred and temporary brachytherapy was provided for 3 days which led to a full remission. A recent magnetic resonance image showed no reoccurrence. But 2 months after the above treatment, $\{$ which one of the 2 treatments "listed above"?\} the patient began to experience significant pain in the throat, the base of the tongue, and jaw causing difficulty in swallowing. It became chronic and progressively worse. At the time of the initial presentation to the pain clinic, the patient scored her pain as 7 of 10 on a " 0 to 10 " pain scale\{did you use the actual Numeric Rating Scale?\} with frequent occurrences of maximal pain (10 of 10). Pain was burning and sharp. The most significantly aggravating factors appeared to be swallowing, coughing, or speaking. Difficulty in swallowing prevented adequate nutrition and the patient reported a loss of
40 pounds despite a normal appetite. Her pain medicine regimen consisted of meperidine hydrochloride $600 \mathrm{mg} / \mathrm{d}$ and lidocaine $2 \%$ daily swish every 4 hours. In addition she was using a nystatin swish and swallow for her oroesophageal fungal infection.

The patient was extremely slender to emaciated but pleasant and cooperative. Her facial appearance was normal in color and appearance except for a mild to moderate fullness over the anterior aspect of the neck and over the area below and above the hyoid bone. Her voice sounded soft and muffled, which the patient reported as a change since her radiation therapy. Neck palpation revealed induration of the inframandibular area but without evidence of adenopathy. External surface motility of the laryngeal structures was normal and deep palpation of the jaws or neck did not reveal significant tenderness. Examination of her oral cavity revealed an edentulous maxilla and mandible. Thrush was seen over the dorsal aspect of the tongue and redness of the tongue and mouth floor, but no lesions on the tongue, gums, inner cheeks, or mouth floor were observed. Her diagnosis was oropharyngeal postradiation neuropathy and mucositis with secondary oral thrush.

A mixture of lidocaine $1 \mathrm{~g}$, diphenhydramine hydrochloride $63 \mathrm{mg}$, nystatin 2.5 megaunits, and gabapentin $10 \mathrm{~g}$ in a total of $100 \mathrm{~mL}$ total volume (final concentration of the mixture: lidocaine $10 \mathrm{mg} / \mathrm{mL}$, diphenhydramine hydrochloride $0.63 \mathrm{mg} / \mathrm{mL}$, nystatin $25,000 \mathrm{U} / \mathrm{mL}$, gabapentin $100 \mathrm{mg} / \mathrm{mL}$ ) was prescribed. The patient was recommended to do swish-and-swallow with this mixture 4 times a day. In addition, due to the ineffectiveness of the maximal dose of meperidine, we switched her to an equianalgesic combination of morphine sulfate sustained release $20 \mathrm{mg}$ by mouth twice a day. Oxycodone hydrochloride $(5 \mathrm{mg})$ and acetaminophen ( $325 \mathrm{mg}$ ) one tablet by mouth every 8 hours as needed were also prescribed.

At the 20-day follow-up visit the pain in her jaw had been completely eliminated. She reported some residual pain due to swallowing. Physical examination 
revealed the thrush on her tongue was improved from the initial presentation and palpation of the mandible and throat failed to reveal significant tenderness. The patient was advised to continue the swish-and-swallow of the lidocaine, diphenhydramine hydrochloride, nystatin, and gabapentin. Morphine sulfate sustained release was decreased to $10 \mathrm{mg}$ by mouth every 12 hours and oxycodone hydrochloride and acetaminophen was continued. The oral cavity biopsy indicated no recurrence of malignancy with subsequent surgery to partially remove/debulk the postradiation scar tissue. A pathology examination of the discarded tissue revealed only necrosis. A significant and consistent improvement in the level of her pain with the swish-and-swallow treatment was maintained. At 2 months follow-up it was possible to discontinue the swish-and-swallow as well as her opioid therapy and the patient's symptoms of sharp burning pain resolved without reoccurrence during the one year follow-up period.

\section{Discussion}

This case describes a patient with severe chronic pain of the mandible and anterior neck following brachytherapy for carcinoma of the base of the tongue that persisted for several months. Standard postradiation treatment failed to provide adequate pain relief, so we added a novel topical mixture of lidocaine, diphenhydramine hydrochloride, nystatin, and gabapentin to a course of oral opioids. This unique regimen provided rapid and effective pain relief, demonstrating that new topical mixtures may be useful in alleviating pain secondary to radiation therapy. The causes of pain secondary to radiotherapy include painful mucosal thinning and ulceration (e.g., oral mucositis, esophagitis, gut pain, perianal pain); myelopathy; fibrosis of the neural plexus (e.g., brachial or lumbar); and peripheral nerve tumors. Among the factors that may determine the occurrence of postradiation pain are the amount of delivered rads, therapy fractionation, prior irradiation, infection, and the degree of tissue vascularization (4).

Mucositis usually appears toward the end of the second week of treatment, reaches a plateau during the fourth week, and may persist for 2 or 3 weeks after the completing treatment (1). Initially, the mucosa of the mouth becomes reddened and swollen, then it becomes covered with a fibrous exudate as the treatment continues. Typically the patient complains of a burning sensation, while the examination of the mouth reveals erythema. Management involves the aggressive use of analgesics (e.g., patient-controlled analgesia) and, eventually, antimicrobial agents (3). This aggressive approach can become counterproductive if the symptoms become unremitting and chronic.

Radiotherapy results in chronic inflammation and subsequent fibrosis of connective tissue that may induce unremitting chronic pain by exerting pressure upon the axons of peripheral nerves $(4,5)$. It is not clear yet what the pathological changes of radiation-induced neuropathy in the peripheral nerves are (5). In every instance, associated occluded or necrotic blood vessels were found. The vascular lesions included acute and chronic vasculitis, fibrinoid necrosis, and telangiectasia.

Opioid therapy alone was not sufficient to treat her symptoms. A combination therapy was needed to facilitate the ability to swallow which was thought to be attainable by the described mixture. A topical ointment mixture of amitriptyline, gabapentin, and lidocaine has previously been used as a treatment for postherpetic neuralgia; diphenhydramine hydrochloride has also been used as a topical treatment for itching in the form of a cream, gel, or spray. To our knowledge, the current report is unique due to the demonstration of a successful nonconventional topical treatment in combination with standard opioid therapy for radiotherapy-induced oral mucositis and peripheral neuropathic pain. Further applications may be useful in treating other patients with this distressing syndrome.

\author{
Nashaat N. Rizk, MD \\ Department of Anesthesiology \\ Division of Pain Medicine \\ University of Pittsburgh Medical Center \\ 3471 Fifth Avenue, Suite 910 \\ Pittsburgh, PA 15213 \\ Victor A. Georgescu, MD \\ Department of Anesthesiology \\ University of Pittsburgh Medical Center \\ Horizon Pain Management \\ Greenville, PA \\ Gaurav Jain, MD \\ Department of Anesthesiology \\ Division of Pain Medicine \\ University of Pittsburgh Medical Center \\ 3471 Fifth Avenue, Suite 910 \\ Pittsburgh, PA 15213 \\ Email: drgauravjain@gmail.com
}




\section{References}

11. Trotti A, Bellm LA, Epstein JB, Frame D, Fuchs HJ, Gwede CK, Komaroff E, Nalysnyk L, Zilberberg MD. Mucositis incidence, severity and associated outcomes in patients with head and neck cancer receiving radiotherapy with or without chemotherapy: A systematic literature review. Radiother Oncol 2003; 66:253-262.

2. Clarkson JE, Worthington HV, Furness
S, McCabe M, Khalid T, Meyer S. Interventions for treating oral mucositis for patients with cancer receiving treatment. Cochrane Database Syst Rev 2010; 4:CDool973.

3. Peterson DE, Bensadoun RJ, Roila F; ESMO Guidelines Working Group. Management of oral and gastrointestinal mucositis: ESMO Clinical Practice
Guidelines. Ann Oncol 2011; 22 Suppl 6:vi78-84.

4. Chapman RC. Pain related to cancer treatment. J Pain Symptom Manage 1988; 3:188-193.

5. Rogers LR. Neurologic complications of radiation. Continuum (Minneap Minn) 2012; 18:343-354.

\section{Tramadol Article Conclusion Troubling}

\section{TO THE EDITOR:}

The meta-analysis by Chung et al (1) is a useful evaluation of the currently utilized drug treatments for chronic low back pain. However, we do not agree with their conclusion that tramadol shows no statistically significant effect on pain relief. The authors included 3 studies (Peloso et al [2], Ruoff et al [3], and Vorsanger et al [4]) in their meta-analysis. Chung et al (1) used the visual analog scale (VAS) pain intensity as a primary efficacy measure for their meta-analysis for the studies by Ruoff et al (3) and Vorsanger et al (4). It seems that for the study by Peloso et al (2) they used the mean and standard deviation (SD) values for change from baseline of the short form McGill Pain Questionnaire/present pain index instead of the VAS pain intensity, which is available as well. In addition, we were unable to deduct from the study by Ruoff et al (3) the SD values for the change from baseline reported by Chung et al (1).

We conducted 2 meta-analyses with datasets D1 and D2. Dataset D1 consists of data reportedly used by Chung et al (1). Dataset D2 differs from D1 in that from the study by Peloso et al (2) the change from baseline in VAS pain intensity has been taken and that the SD value ${ }^{S} C$ for the study by Ruoff et al (3) was calculated in the same way as it was calculated for the other 2 studies as follows (Table 1 ):

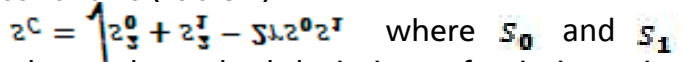
are the observed standard deviations of pain intensity scores at baseline and end-of-treatment, respectively, and $r$ is the correlation between pain intensity scores at baseline and end-of-treatment. Since this correlation is not reported, we have assumed $r=0.8$ in all studies following what Chung et al (1) presumably did for the study of Vorsanger et al (4). Peloso et al (2) and Ruoff et al (3) did not report $S_{1}$; therefore we have assumed that $S_{1}=s_{0}$.

Our results for dataset D1 are identical to those of Chung et al (1). The results for dataset D2 showed a statistically significant difference (estimated overall effect $-1.18 ; 95 \%$ confidence interval -1.65 to $-0.71 ; P<$ 0.0001 ) between tramadol and placebo (Table 2 ).

We conclude that there is persuasive evidence for a benefit of tramadol alone or in fixed dose combination with acetaminophen in the management of chronic low back pain. This is important to report because, in contrast to nonsteroidal anti-inflammatory drugs and Cox II inhibitors, long-term use of tramadol is not associated with an increased risk of gastrointestinal, renal, and cardiovascular organ damage and, in contrast to strong opioids, the risk of respiratory depression, addiction, and abuse is lower.

Juan J Abellan, PhD

Trial Biostatistician

Global Biometrics

Grünenthal $\mathrm{GmbH}$

Zieglerstrasse 6

52078 Aachen

E-mail: Juan.Abellan-Andres@grunenthal.com

Johannes CG van Niel, PhD

International Clinical Lead Mature Products

Global Clinical Development Strategy 